\title{
Controversies in the role of radiotherapy in pleural mesothelioma
}

\author{
Gerard G. Hanna $^{1,2} \wedge$, Thomas John ${ }^{2,3}$, David L. Ball ${ }^{1,2}$ \\ ${ }^{1}$ Department of Radiation Oncology, Peter MacCallum Cancer Centre, Melbourne, Victoria, Australia; ${ }^{2}$ Sir Peter MacCallum Department of Oncology, \\ University of Melbourne, Victoria, Australia; ${ }^{3}$ Department of Medical Oncology, Peter MacCallum Cancer Centre, Melbourne, Victoria, Australia \\ Contributions: (I) Conception and design: GG Hanna, DL Ball; (II) Administrative support: GG Hanna; (III) Provision of study materials or patients: \\ GG Hanna, DL Ball; (IV) Collection and assembly of data: All authors; (V) Data analysis and interpretation: All authors; (VI) Manuscript writing: All \\ authors; (VII) Final approval of manuscript: All authors. \\ Correspondence to: Prof. Gerard G. Hanna. Department of Radiation Oncology, Peter MacCallum Cancer Centre, 305 Grattan Street, Melbourne, \\ Victoria 3000, Australia. Email: gerry.hanna@petermac.org.
}

\begin{abstract}
Malignant pleural mesothelioma is an uncommon thoracic cancer with a relatively poor outcome, which has only seen modest improvements when compared to non-small cell lung cancer. The mainstays of treatment have been surgery and systemic therapy, with radiation reserved for palliation or as an adjunct. However, there is re-emergent interest in the use of radiotherapy in the treatment of mesothelioma, given recent technical advances in radiotherapy delivery which permit increased treatment accuracy. This overview article reviews the radiobiology of the mesothelioma and whether or not mesothelioma is an inherently radioresistant cancer and the potential impact that hypofractionation may have on different histological subtypes in mesothelioma. This overview also considers the role of radiation in palliation, as adjunct to surgical resection and as adjunct to pleural tract procedures. In particular we review the growing evidence that pleural tract or port site adjuvant radiotherapy provides no clinical benefit. This overview will also consider potential emerging therapeutic strategies such as pre-operative short course hypofractionated radiotherapy. The role of novel radiotherapy techniques such as stereotactic ablative radiotherapy, image guided radiotherapy, proton therapy and the potential role of radiotherapy as an immune stimulating agent in combination of immunotherapy, will also be discussed. Finally, given the many unanswered questions, this review discusses some of the emerging and ongoing clinical trials of radiotherapy in the treatment of mesothelioma.
\end{abstract}

Keywords: Mesothelioma; radiotherapy; palliative radiotherapy; hemithoracic radiotherapy; radiation therapy

Submitted Apr 20, 2020. Accepted for publication Jul 14, 2020.

doi: $10.21037 /$ tlcr-20-583

View this article at: http://dx.doi.org/10.21037/tlcr-20-583

\section{Introduction}

Malignant pleural mesothelioma, although an uncommon thoracic malignancy, remains a cancer of poor outcome with a significant burden of morbidity for those affected. Incidence rates are still on the rise in many countries globally, reflecting the ongoing risk from the main aetiological agent, asbestos. The use of radiotherapy in the treatment of patients with mesothelioma has largely been adjuvant or palliative.
Delivering radical radiotherapy has been hampered by the ability to safely avoid key organs at risk, but principally the lungs. Three systematic reviews (1-3) and a Cochrane review of the role of radiotherapy in mesothelioma in the last 15 years all conclude there is still a paucity of highlevel evidence to guide the most effective use (if any) of radiotherapy for this disease (4). In this article we will attempt to address the most controversial contemporary questions and make suggestions for further lines of investigation.

\footnotetext{
^ ORCID: 0000-0003-1003-5138.
} 


\section{Is mesothelioma an inherently radioresistant} cancer?

In considering the role of radiotherapy in mesothelioma, the first question to consider is if mesothelioma is responsive to radiotherapy or radioresistant. Clinical evidence of response to radiotherapy using RECIST criteria is essentially non-existent as the sheet-like pattern of growth seen in most cases of mesothelioma does not lend itself to the application of the classical RECIST method (5). In addition, radiotherapy has been most commonly used in the postoperative adjuvant setting or for pain relief; in both cases accurate measurement of disease volume (and therefore response) using modern 3D CT segmentation software can be challenging. Even so, there is an old perception that mesothelioma is an inherently radioresistant cancer which requires high doses to produce a response. For example, Gordon et al. observed that high doses of "conventional radiotherapy" did not control 1-2 mm nodules left after surgery (6). In contrast, case reports of modest doses of palliative radiotherapy have delivered significant clinical benefit (7). Furthermore, the SYSTEMS study reported by MacLeod et al., the authors report a clear symptomatic response to $20 \mathrm{~Gy}$ in 5 fractions, widely considered a relatively low dose of palliative radiotherapy (8).

One consideration in the radiation response of a given tumour to radiotherapy is not just the total overall dose, but the response as the dose per fraction of radiotherapy changes. This is estimated by the radiobiological measure of alpha/beta ratio $(\alpha / \beta)$. Typically, tumours that are rapidly proliferating (e.g., lung squamous cell non-small cell carcinoma) have high $\alpha / \beta$ ratios, whilst more slowly proliferating tumours (e.g., prostate carcinoma) have low $\alpha / \beta$ ratios. Given that mesothelioma has a non-squamous histology, a low proliferation index, and suggested radioresistance, it is likely that mesothelioma has a low $\alpha / \beta$ ratio, inferring that mesothelioma is more sensitive to increases in dose per fraction. This would suggest that mesothelioma might respond better to increasing dose per fraction. In a retrospective study of 227 radiotherapy treatments to 189 patients in the Netherlands, patients treated with a 4-Gy per fraction palliative regimen had a higher local response to treatment compared to those receiving fractions of less than 4 Gy (50\% vs. 39\%) (9). Further hypofractionation is currently being tested in the SYSTEMS-2 study which is comparing 20 Gy in 5 fractions to $36 \mathrm{~Gy}$ in 6 fractions of palliative radiotherapy (10).

Beyond conventional radiation dose response and the impact of hypofractionation, there is a paucity of information on the effect of systemic therapy as a radiosensitizer in the treatment of mesothelioma. Beyond the classical combination of a cytotoxic agent with radiation, other potential combinations could include the combination of DNA damage repair agents or immune modulating agents with radiation as is being considered in other primary tumour sites (11). Certainly, there is a clear need to address the absence of pre-clinical and clinical data regarding the radiosensitivity of mesothelioma to either radiation alone or radiation in combination with systemic therapy.

\section{Radiotherapy in the palliation of mesothelioma}

As described above the use of palliative radiotherapy in mesothelioma has been shown to be beneficial with symptom improvement. Given the insidiously invasive nature of mesothelioma, using radiotherapy to palliate local invasion is logical. The SYSTEMS study provides the most contemporary cohort and used a dose of $20 \mathrm{~Gy}$ in 5 fractions. Both 2-dimensional and 3-dimensional planning were used to plan treatment. However, one challenge in delivering palliative radiotherapy in mesothelioma is identifying the appropriate target volume. Commonly chest wall pain may be difficult to localise within diffuse disease throughout the pleural cavity, thus making precise targeting of the symptomatic site more an art than a science. Even in the era of 3-dimensional radiotherapy, most palliative radiation fields in mesothelioma are largely based on the localisation of symptoms, correlated with imaging as best possible.

As discussed above the dose response of mesothelioma to radiation has yet to be fully determined. In terms of recommended radiotherapy dose and fraction regimens, the recent American Society of Clinical Oncology clinical practice guideline and expert opinion from the National Cancer Institute Thoracic Malignancy Steering Committee, International Association for the Study of Lung Cancer, and Mesothelioma Applied Research Foundation all recommend an 8 Gy single fraction for the palliation of uncomplicated metastases $(12,13)$.

\section{Port site irradiation or prophylactic radiotherapy for procedure track metastases-is this now an obsolete procedure?}

Histological diagnostic confirmation of mesothelioma is usually obtained via an invasive procedure such as CT-guided needle biopsy, thoracoscopy, video-assisted thoracoscopic surgery (VATs) or thoracotomy. These 


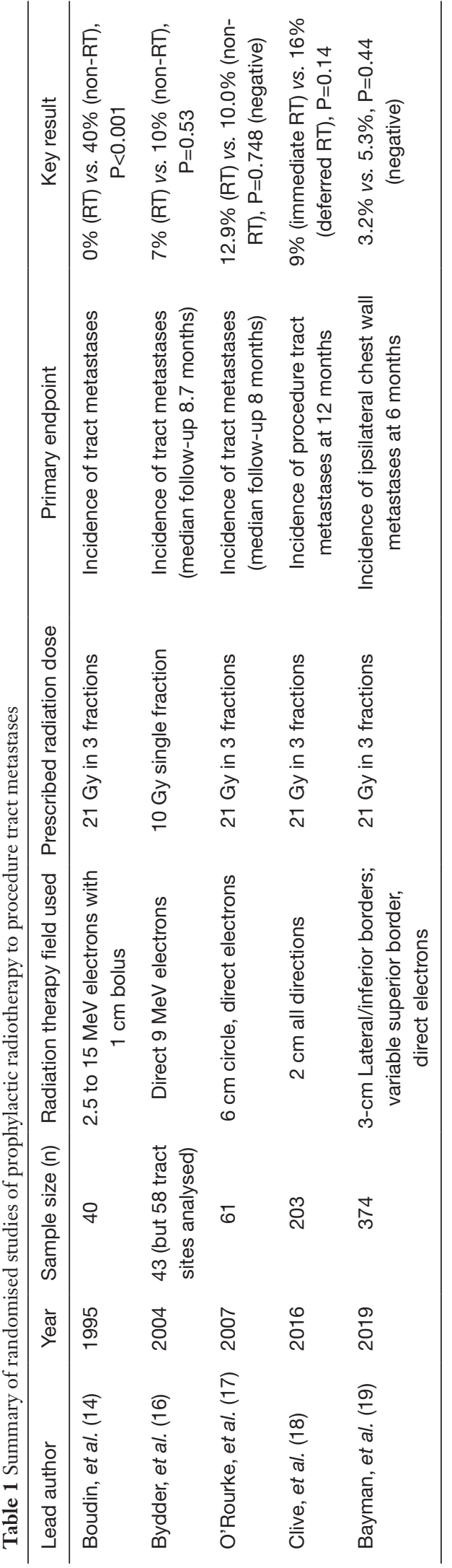

procedures along with pleural drainage can lead to metastatic seeding along the track for the procedure through the chest wall in up to $50 \%$ of patients following the procedure $(14,15)$. Port site irradiation or prophylactic radiotherapy for procedure track metastases describes administering an adjuvant radiotherapy dose to the biopsy site of chest drain external wound site to reduce the risk of metastatic seeding of mesothelioma along the drain site. Following the results of an early randomised study which reported a clear benefit to offering prophylactic radiotherapy, it became established practice to offer and adjuvant dose of radiation therapy to the "port site" (14). Radiation therapy planning was simple, usually undertaken with a clinical mark up to encompass the area around the port site with a margin of $2-3 \mathrm{~cm}$ and delivered with a direct electron field to a dose of 21 Gy in 3 daily fractions. Toxicity was usually mild, confined mainly to skin erythema and chest wall pain. In spite of the positive results of the Boutin et al. study, the positive benefit of port site radiotherapy was not observed in 4 subsequent randomised studies. The details of all 5 randomised studies are listed in Table 1 (16-19). The most recent of these is the PIT study, reported by Bayman et al. (19). This UK study randomised 374 patients to port site radiotherapy versus observation. Radiation therapy fields used in this study attempted to more comprehensively cover the procedure track than the techniques used in previous studies. In spite of these and other trial design considerations, no benefit to port site radiotherapy was observed. It is worth noting that the rates procedure track metastases in both arms of the PIT study of were much lower than those previously reported by Boutin et al. in 1995. One potential explanation might be differential rates of systemic therapy usage in the treatment of mesothelioma. However, it is now clear that there is no role for prophylactic radiation therapy in the treatment of procedure tracts in mesothelioma. It should be borne in mind that treatment of established port site recurrence may be just as effective as prophylaxis, recognising that port site recurrence is in most instances "the tip of the iceberg" in patients with mesothelioma.

\section{Will the question of a benefit of adjuvant radical hemithoracic radiotherapy in conjunction with surgery ever be resolved?}

There is no more technically demanding challenge in radiation oncology than safely giving a radical dose of radiotherapy (generally considered 50 Gy or higher) to the 
whole hemithorax with the intention of covering the pleural reflections and any disease extension into the mediastinum and chest wall. That it is usually done in a patient who has already undergone either an extrapleural pneumonectomy or pleurectomy/decortication, ensures that it can only be offered to the fittest and most motivated patients since both operations are associated with significant morbidity and mortality.

The infiltrative and sheet-like pattern of growth over the pleural surface that characterises mesothelioma poses special problems for the surgeon wishing to achieve an R0 resection except in the uncommon setting where it is very well localised. Incomplete resection and local failure rates in most patients after surgery are understandably high. There is still scepticism and divided opinion within the thoracic surgical community about the value of any form of aggressive surgery for pleural mesothelioma. A randomised trial (MARS) designed to test the feasibility of randomising 50 patients in one year to extrapleural pneumonectomy followed by 3D conformal radiotherapy (54 Gy) versus no pneumonectomy did not reach its feasibility endpoint (20). In this, the only randomised trial in which the effectiveness of extrapleural pneumonectomy has been compared with no pneumonectomy, there was no indication of a benefit for pneumonectomy in a multimodality setting, and even a suggestion that it may have been harmful. One hundred and twelve patients were registered, but only 50 patients reached the point of randomisation. Median survival for the pneumonectomy patients was 14.4 months versus 19.5 months for no pneumonectomy, with a hazard ratio of 1.90 (95\% CI: 0.92-3.93). In fact, the randomisation was between extrapleural pneumonectomy plus hemithoracic radiotherapy (54 Gy in 30 fractions using a 3D conformal technique) versus standard oncological care, so it is possible that any differences between arms might have been due in part to the radiotherapy component. Of the 24 patients randomised to pneumonectomy, only eight (33\%) went on to have radical radiotherapy as required by the protocol. Five (19\%) of the 26 patients randomised to no pneumonectomy but had some form of radiotherapy but further details are not provided.

The effect of post pneumonectomy radical radiotherapy was tested in a randomised phase II trial of the Swiss Group for Clinical Cancer Research (SAKK 17/04) (21). Patients with pleural mesothelioma who had achieved complete resection as a result of induction chemotherapy and extrapleural pneumonectomy were randomised to radical hemithoracic radiotherapy (55.9 Gy median) or observation.
The trial was closed prematurely because of slow accrual with 54 patients randomised to radiotherapy or observation. There was no significant difference between arms in the primary endpoint, local relapse free survival after surgery, with a median of 7.6 months (95\% CI: $4.5-10.7)$ in the no radiotherapy group versus 9.4 months (95\% CI: 6.5-11.9) in the radiotherapy arm. Similarly, median survival measured from registration (before chemotherapy or surgery) was 20.8 months in patients randomised to no radiotherapy versus 19.3 months for radiotherapy. Interestingly there were no changes in overall quality of life in both groups up to 14 weeks after registration. There was only one grade 5 toxicity (pneumonitis) in the radiotherapy arm. The authors concluded that the addition of hemithoracic radiotherapy imposed an additional treatment burden without patient benefit. These conclusions were questioned because of the lack of statistical power due to low numbers with only 54 patients eventually randomised from the 151 patients who had been registered for chemotherapy and surgery in the first part of the trial (22). The high attrition rate prior to radiotherapy randomisation due to disease progression, unresectability, postoperative mortality (4\% at 30 days, $8 \%$ at 60 days) and patient refusal are well documented in the CONSORT diagram. Furthermore, there was a lack of detail regarding radiotherapy planning, delivery and doses used. The methods used to detect and define locoregional recurrence, notoriously difficult in the post-pneumonectomy setting, were also not described. In an updated analysis the SAKK trialists reported the patterns of failure with relation to the radiotherapy field. Interestingly, of the 27 patients randomised to radiotherapy, only 1 patient had a local recurrence, suggesting local efficacy of radiotherapy, but overall futility as a result of failure to control out of field disease (23).

What can we learn from the MARS and SAKK trials? Firstly, in both trials fewer than half the patients registered were able to complete treatment according to randomisation. Secondly, extrapleural pneumonectomy is a high-risk procedure with a significant mortality and questionable therapeutic benefit. Finally, post pneumonectomy hemithoracic radiotherapy is feasible with a low mortality in patients who were able to receive the prescribed radiation.

Might a lesser operation than pneumonectomy be less harmful without loss of effect? In a meta-analysis, pleurectomy/decortication was associated with a lower risk of 30-day mortality compared with extrapleural pneumonectomy, with no overall survival disadvantage (24). 


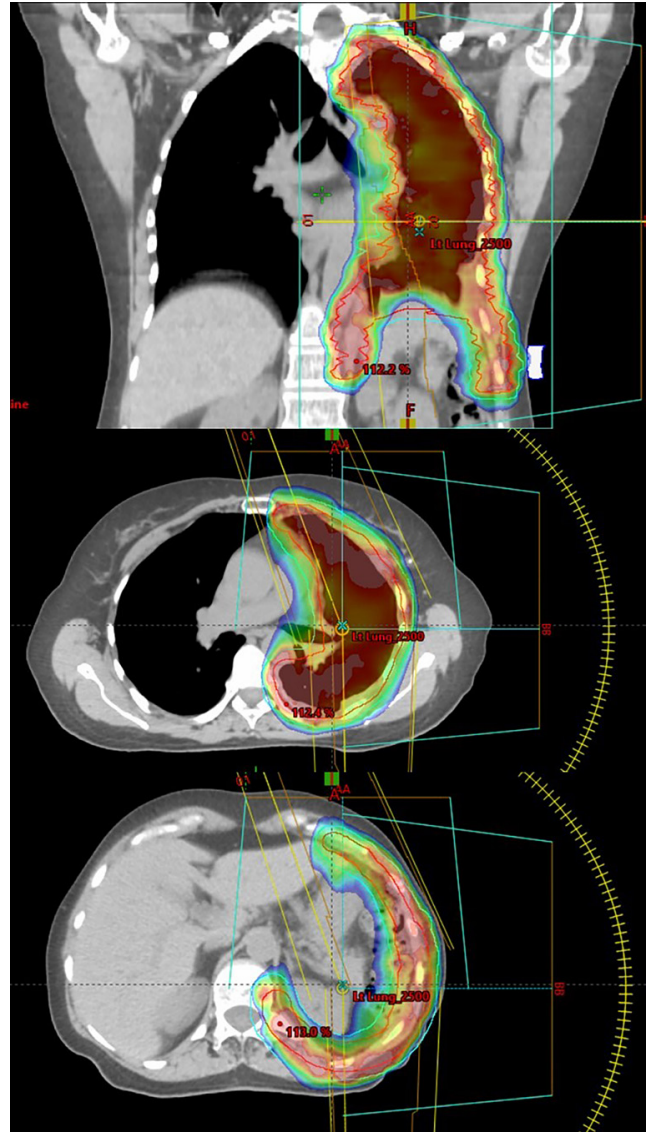

Figure 1 Representative coronal and transaxial slices of a hemithoracic volumetric arc therapy (VMAT) plan. The GTV is denoted by the red line, the planning target volume (PTV) by the blue line and the colour wash displays the dose intensity from $90 \%$ to $107 \%$ of the prescribed dose. By using this intensity modulated radiotherapy (IMRT), it is possible to curve the dose around organs at risk such as the intra-abdominal organs.

Does a shift in practice to pleurectomy have implications for adjuvant radiotherapy as a tool to reduce local failure? The presence of functioning lung within the irradiated volume is a disadvantage because of a higher risk of pneumonitis and the detrimental haemodynamics of shunting within an irradiated lung compared with no lung at all. The clinical target volume at risk is also more complex because of the possibility of recurrence in the unstripped visceral pleura of the fissures deep within the lung. Addressing this issue of the complex target volume delineation in this setting, Gomez et al., set out clear contouring guidelines in their expert opinion paper (13). In this they recommend covering the costophrenic, costodiaphragmatic and cardiophrenic angles to ensure coverage of the entirety of the pleural recesses as well as a number of other planning recommendations.

Advanced radiotherapy techniques have now evolved to the point that the pleural envelope at risk can be irradiated to radical doses with relative sparing of the underlying lung using intensity modulated radiotherapy (IMRT). An example of an IMRT type plan, also known as volumetric arc therapy (VMAT) is shown in Figure 1. A multicentre phase II US trial demonstrated the safety of pleural IMRT (IMPRINT) in patients with two intact lungs in which the planned dose of radiotherapy after chemotherapy with or without pleurectomy was $50.4 \mathrm{~Gy}$, with a guideline for combined mean lung dose $\leq 21$ Gy and combined lung volume receiving $20 \mathrm{~Gy}\left(\mathrm{~V}_{20 \mathrm{~Gy}}\right)$ of $\leq 37-40 \%$ (25). The primary endpoint was the rate of grade 3 or greater pneumonitis. Forty-five patients were enrolled, but only 27 patients were evaluable, mainly because of disease progression or refusal of treatment. There were only two cases (7\%) of grade 3 pneumonitis, although not all patients appear to have received the planned dose of radiotherapy. The median value of combined mean lung dose was $20.9 \mathrm{~Gy}$, with a range of 16.2-24.2 Gy. The median value of the combined lung $\mathrm{V}_{20 \mathrm{~Gy}}$ was $38.8 \%$ with a range $21.6-43.3 \%$. The main toxicity was severe protracted fatigue, which is familiar to any clinician who has managed patients with multimodality therapy for mesothelioma which includes at the end a demanding 6-week course of radiotherapy.

In part, to spare the patient this ordeal, the Toronto group developed an accelerated hypofractionated induction radiotherapy schedule which was given over a week to the hemithorax, followed within a week by extrapleural pneumonectomy, a protocol they termed Surgery for Mesothelioma after Radiation Therapy (SMART) (26). Radiotherapy using the SMART schedule was given using IMRT to the whole of the hemithorax to include the pleura and intervention tract sites. The dose was $25 \mathrm{~Gy}$ in five daily fractions with a concomitant 5 Gy boost to the gross tumour volume. Such a dose to the intact lung would be expected to produce a high rate of fatal pneumonitis, but this problem is avoided with removal of the lung soon after completion of radiotherapy. Patients with lymph node involvement were given adjuvant chemotherapy. The other rationale for the neoadjuvant approach was the potential for reduction of dissemination of viable tumour cells at surgery.

Of 256 patients assessed for a phase II study, only 62 were suitable, with advanced disease, comorbidity and patient refusal major reasons for exclusion (27). All patients completed radiotherapy as planned. All but 1 
patient underwent resection and reconstruction of the diaphragm, and all but 4 patients underwent resection and reconstruction of the pericardium. No patient died within 30 days of surgery but there were 3 (4.8\%) treatment-related deaths. The median overall survival was 36 months. Among patients with cT1-3N0M0 disease $(\mathrm{n}=56)$, the median disease free survival was estimated to be 47 months and overall survival was 51 months in patients with the epithelial subtype, compared to only 8 and 10 months, respectively, in patients with the biphasic subtype, suggesting a differential response to hypofractionated radiotherapy between the epithelial and biphasic subtype.

Of critical importance, the use of radiation as an adjunct to surgery requires very careful patient selection. In SMART, the median age was young at 64 and patients with epithelial histology and less advanced disease achieved the best survival. Very careful staging, subsequent exclusion of patients with non-epthelioid histology multiple comorbidities and careful functional assessments were required to achieve the impressive results reported. Despite this however, 29\% developed > grade 3 complications, including three treatment related deaths. It is clear from the evidence presented that the role of adjuvant radiotherapy in patients having surgery for pleural mesothelioma is uncertain. Because it is associated with significant morbidity and places considerable demands on patients and busy departments with limited resources, its use (as with radical surgery) should be restricted to the clinical trial setting.

The outcomes of the IMPRINT study have formed the basis for a trial of pleurectomy/decortication and chemotherapy followed by randomisation to observation or IMRT under the leadership of NRG Oncology (NRGLU006) (28). This ambitious trial plans to randomise 150 patients to 45-50.4 Gy over six weeks using photons or protons. The statistical calculations are based on the hypothesis that the addition of radiotherapy will increase median survival from 12 to 20 months (HR 0.6). The recruitment of the required number of eligible patients, patient compliance with a demanding multimodality treatment program, and a hazard ratio of 0.6 will be a remarkable achievement if the difficulties encountered and the results observed in the MARS and SAKK trials are anything to go by. A retrospective analysis of real world data from the US National Cancer Database 2004 to 2011 which compared survival with and without adjuvant radiotherapy following surgery for mesothelioma did find a benefit for radiotherapy in patients with stage I and II disease (HR 0.52, 95\% CI: 0.29-0.95), but not for patients with stage III (HR
1.24, 95\% CI: 0.90-1.71) or IV disease (HR 0.85, 95\% CI: 0.49-1.48) after adjustment for other prognostic factors (29). However, patients with stage I/II mesothelioma made up only $19 \%$ of the total, with a mere $4 \%$ of these having adjuvant radiotherapy, further emphasising the size of the task ahead of the NRG-LU006 investigators. One design feature of note with the NRG-LU006 study is the use of either photons or protons. Whilst protons have a theoretical advantage of potentially sparing more normal tissue that photons, only small cases series exist reporting outcomes for protons in mesothelioma (30).

The follow-on trial from SMART is the SMARTER study (31). As with SMART it is a study of neoadjuvant radiotherapy before surgery investigating dose escalation to the GTV for its possible immunostimulatory properties. The primary endpoint is the maximum tolerated dose. The planned accrual is 18 patients. The question remains, is there room for two separate adjuvant radiotherapy trials for pleural mesothelioma in North America (or for that matter, the world)? Will these trials provide practice changing evidence within a realistic timeframe? Or before other interventions such as immunotherapy prove more effective and less morbid? The planned completion date for NRG-LU006 is 2030. Would a smaller randomised phase II "pick the winner" trial comparing IMPRINT with SMART be a more feasible and interesting project providing an option for clinicians and patients who cannot accept minimal or no treatment as an option?

\section{Remaining unanswered questions for radiotherapy in mesothelioma}

So how best to plan and deliver radiotherapy in mesothelioma? In terms of planning and simulation there is some suggestive evidence that PET/CT may better guide target volume delineation as compared to CT alone (32). In a planning study of 13 patients, PET/CT was shown to reduce the risk of geographic miss.

As mentioned above advanced radiotherapy techniques such as IMRT, VMAT and proton therapy permit better radiotherapy dose delivery to the tumour whilst better sparing surrounding health normal tissues. What has not been tested is the therapeutic gain of using such advanced radiotherapy techniques for the large fields used for some indications in mesothelioma. Allen et al. reported outcomes for 13 patients receiving IMRT following extra-pleural pneumonectomy (33). Of the 6 patients who developed fatal pneumonitis in the remaining lung, the V20 was numerically higher leading the authors to recommend stringent dose 
constrains in this clinical setting. In patients with a single intact lung, they recommended keeping the MLD to $<8.5$ Gy, the V20 to $<10 \%$, the V10 to $<55 \%$ and the V5 to $<60 \%$ as well as arranging the IMRT beams to avoid the remaining lung entirely. It is however very unlikely that conducting a study such as IMRT $v$ s. VMAT or protons versus photons, would ever be feasible. Furthermore, given the difficulty of obtaining similar supporting evidence of any benefit of using proton therapy in other more common disease entities, such as non-small cell lung cancer, a similar trial in mesothelioma remains highly unlikely (34).

As seen in both the MARS and SMART study, the extensive surgical resection employed in EPP has an associated morality rate and morbidity rate. But what about using radiotherapy as the primary treatment alone or in combination with systemic therapy as radical therapy? At present, no such study exists, but this may be an attractive option for patients with borderline operability. Any study addressing this would have to employ advance radiotherapy techniques as described above, consider how best to sequence the systemic therapy and radiotherapy combination, and consider the use of novel agents such immune modulating agents or DNA damage repair inhibitors. Whilst many trials are investigating the role of immunotherapy in mesothelioma at present, the results of immunotherapy alone following initial chemotherapy are provisionally disappointing when compared to chemotherapy. One potential avenue of synergy is the combination of immunotherapy and radiotherapy, with the potential to enhance both the adscopal and abscopal effect $(35,36)$. In addition to the directly cytotoxic effect of radiation sterilizing the surgical field and thus limiting the risk of spillage at the time of surgery, one postulated underlying mechanism for the excellent results seen with the SMART protocol, is that radiotherapy is immunostimulatory and thus enhances an abscopal effect. This potential combination of immunotherapy and radiotherapy is being investigated by a number of clinical trials in set-up. The MESO-PRIME study plans to investigate if stereotactic ablative radiotherapy (SABR) when used a priming dose can enhance the efficacy of pembrolizumab in mesothelioma. The MESO-PRIME investigators plan to deliver an immunostimulatory dose of SABR to a suitable part of the tumor, but not to all areas of disease (37). Similarly, a Phase 1 study seeks to examine the safety of the combination of atezolizumab, pemetrexed, cisplatin, and surgery with or without radiotherapy patients with stage I-III mesothelioma (38).
Finally, the role of SABR in the treatment of recurrent or metastatic mesothelioma has not been well reported. Schröder et al. report a series of 21 patients treated with SABR for oligorecurrence of malignant pleural mesothelioma. Only 1 patient experienced a grade 3 toxicity and the 12 -month local control rate was $73.5 \%$, suggesting both feasibility and efficacy for this approach (39). It is likely that SABR will be become more widely used in the treatment of oligometastatic and progressive disease.

\section{Conclusions}

The role of radiotherapy in malignant mesothelioma remains uncertain in a number of clinical scenarios. Radiotherapy should be used to palliative local symptoms refractory to symptomatic measures or systemic therapy. Radiotherapy should not be used in the prophylactic treatment of procedure related tracts. The role of radiotherapy as an adjunct to surgical resection remains uncertain and results of two prospective randomised studies are awaited. However, the convenience, low rates of toxicity and promising clinical outcomes suggest that hypofractionated pre-operative short course radiotherapy is a potential therapeutic approach, but further evidence is needed.

\section{Acknowledgments}

We thank Smaro Lazarakis, Librarian at the Peter MacCallum Cancer Centre, for her help in collating the manuscripts included in this review.

Funding: None.

\section{Footnote}

Provenance and Peer Review: This article was commissioned by the Guest Editors (Jacek Jassem and Rafal Dziadziuszko) for the focused issue "Radiotherapy in thoracic malignancies" published in Translational Lung Cancer Research. The article was sent for external peer review organized by the Guest Editors and the editorial office.

Peer Review File: Available at http://dx.doi.org/10.21037/ tlcr-20-583

Conflicts of Interest: All authors have completed the ICMJE uniform disclosure form (available at http://dx.doi. org/10.21037/tlcr-20-583). The focused issue "Radiotherapy 
in thoracic malignancies" was commissioned by the editorial office without any funding or sponsorship. GGH reports personal fees from AstraZeneca, outside the submitted work. TJ reports personal fees from Astra Zeneca, personal fees from BMS, personal fees from Novartis, personal fees from Merck, personal fees from MSD, personal fees from Amgen, personal fees from Roche, outside the submitted work. DLB reports personal fees from Astra Zeneca, outside the submitted work. The authors have no other conflicts of interest to declare.

Ethical Statement: The authors are accountable for all aspects of the work in ensuring that questions related to the accuracy or integrity of any part of the work are appropriately investigated and resolved.

Open Access Statement: This is an Open Access article distributed in accordance with the Creative Commons Attribution-NonCommercial-NoDerivs 4.0 International License (CC BY-NC-ND 4.0), which permits the noncommercial replication and distribution of the article with the strict proviso that no changes or edits are made and the original work is properly cited (including links to both the formal publication through the relevant DOI and the license). See: https://creativecommons.org/licenses/by-nc-nd/4.0/.

\section{References}

1. Ung YC, Yu E, Falkson C, et al. The role of radiation therapy in malignant pleural mesothelioma: a systematic review. Radiother Oncol 2006;80:13-8.

2. Price $\mathrm{A}$. What is the role of radiotherapy in malignant pleural mesothelioma? Oncologist 2011;16:359-65.

3. Ashton M, O'Rourke N, Currie S, et al. The role of radical radiotherapy in the management of malignant pleural mesothelioma: A systematic review. Radiother Oncol 2017;125:1-12.

4. Chapman E, Berenstein EG, Diéguez M, et al. Radiotherapy for malignant pleural mesothelioma. Cochrane Database Syst Rev 2006;(3):CD003880.

5. Byrne MJ, Nowak AK. Modified RECIST criteria for assessment of response in malignant pleural mesothelioma. Ann Oncol 2004;15:257-60.

6. Gordon W Jr, Antman KH, Greenberger JS, et al. Radiation therapy in the management of patients with mesothelioma. Int J Radiat Oncol Biol Phys 1982;8:19-25.

7. Ball DL, Cruickshank DG. Available online: https://journals.lww.com/amjclinicaloncology/
Abstract/1990/02000/The_Treatment_of_Malignant_ Mesothelioma_of_the.2.aspx

8. MacLeod N, Chalmers A, O'Rourke N, et al. Radiotherapy Useful for Treating Pain in Mesothelioma?: A Phase II Trial. J Thorac Oncol 2015;10:944-50.

9. de Graaf-Strukowska L, van der Zee J, van Putten W, et al. Factors influencing the outcome of radiotherapy in malignant mesothelioma of the pleura--a single-institution experience with 189 patients. Int J Radiat Oncol Biol Phys 1999;43:511-6.

10. Ashton M, O'Rourke N, Macleod N, et al. SYSTEMS-2: A randomised phase II study of radiotherapy dose escalation for pain control in malignant pleural mesothelioma. Clin Transl Radiat Oncol 2017;8:45-49.

11. Harrow S, Hanna GG, Faivre-Finn C, et al. The Challenges Faced in Developing Novel Drug Radiation Combinations in Non-small Cell Lung Cancer. Clin Oncol (R Coll Radiol) 2016;28:720-5.

12. Kindler HL, Ismaila N, Armato SG 3rd, et al. Treatment of Malignant Pleural Mesothelioma: American Society of Clinical Oncology Clinical Practice Guideline. J Clin Oncol 2018;36:1343-73.

13. Gomez DR, Rimner A, Simone CB 2nd, et al. The Use of Radiation Therapy for the Treatment of Malignant Pleural Mesothelioma: Expert Opinion from the National Cancer Institute Thoracic Malignancy Steering Committee, International Association for the Study of Lung Cancer, and Mesothelioma Applied Research Foundation. J Thorac Oncol 2019;14:1172-83.

14. Boutin C, Rey F, Viallat JR. Prevention of malignant seeding after invasive diagnostic procedures in patients with pleural mesothelioma. A randomized trial of local radiotherapy. Chest 1995;108:754-8.

15. Attanoos RL, Gibbs AR. The pathology associated with therapeutic procedures in malignant mesothelioma. Histopathology 2004;45:393-7.

16. Bydder S, Phillips M, Joseph DJ, et al. A randomised trial of single-dose radiotherapy to prevent procedure tract metastasis by malignant mesothelioma. Br J Cancer 2004;91:9-10.

17. O'Rourke N, Garcia JC, Paul J, et al. A randomised controlled trial of intervention site radiotherapy in malignant pleural mesothelioma. Radiother Oncol 2007;84:18-22.

18. Clive AO, Taylor H, Dobson L, et al. Prophylactic radiotherapy for the prevention of procedure-tract metastases after surgical and large-bore pleural procedures in malignant pleural mesothelioma (SMART): A multicentre, open-label, phase 3, randomised controlled trial. Lancet Oncol 2016;17:1094-104. 
19. Bayman N, Appel W, Ashcroft L, et al. Prophylactic Irradiation of Tracts in Patients With Malignant Pleural Mesothelioma: An Open-Label, Multicenter, Phase III Randomized Trial. J Clin Oncol 2019;37:1200-8.

20. Treasure T, Lang-Lazdunski L, Waller D, et al. Extra-pleural pneumonectomy versus no extra-pleural pneumonectomy (standard oncological management allowed) for patients with malignant pleural mesothelioma: clinical outcomes of the Mesothelioma and Radical Surgery (MARS) randomised feasibility study. Lancet Oncol 2011;12:763-72.

21. Stahel RA, Riesterer O, Xyrafas A, et al. Neoadjuvant chemotherapy and extrapleural pneumonectomy of malignant pleural mesothelioma with or without hemithoracic radiotherapy (SAKK 17/04): a randomised, international, multicentre phase 2 trial. Lancet Oncol 2015;16:1651-8.

22. Rimner A, Simone CB 2nd, Zauderer MG, et al. Hemithoracic radiotherapy for mesothelioma: lack of benefit or lack of statistical power? Lancet Oncol 2016;17:e43-4.

23. Riesterer O, Ciernik IF, Stahel RA, et al. Pattern of failure after adjuvant radiotherapy following extrapleural pneumonectomy of pleural mesothelioma in the SAKK 17/04 trial. Radiother Oncol 2019;138:121-5.

24. Taioli E, Wolf AS, Flores RM. Meta-analysis of survival after pleurectomy decortication versus extrapleural pneumonectomy in mesothelioma. Ann Thorac Surg 2015;99:472-80.

25. Rimner A, Zauderer MG, Gomez DR, et al. Phase II Study of Hemithoracic Intensity-Modulated Pleural Radiation Therapy (IMPRINT) As Part of Lung-Sparing Multimodality Therapy in Patients With Malignant Pleural Mesothelioma. J Clin Oncol 2016;34:2761-8.

26. Cho BC, Feld R, Leighl N, et al. A feasibility study evaluating Surgery for Mesothelioma After Radiation Therapy: the "SMART" approach for resectable malignant pleural mesothelioma. J Thorac Oncol 2014;9:397-402.

27. de Perrot M, Feld R, Leighl NB, et al. Accelerated hemithoracic radiation followed by extrapleural pneumonectomy for malignant pleural mesothelioma. J Thorac Cardiovasc Surg 2016;151:468-73.

28. ClinicalTrials.gov. Testing the Addition of Targeted Radiation Therapy to Surgery and the Usual Chemotherapy Treatment (Pemetrexed and Cisplatin [or Carboplatin]) for Stage I-IIIA Malignant Pleural Mesothelioma. Available online: https://clinicaltrials.gov/ ct2/show/NCT04158141

29. Nelson DB, Rice DC, Mitchell KG, et al. Defining the role of adjuvant radiotherapy for malignant pleural mesothelioma: a propensity-matched landmark analysis of the National
Cancer Database. J Thorac Dis 2019;11:1269-78.

30. Badiyan SN, Molitoris JK, Zhu M, et al. Proton beam therapy for malignant pleural mesothelioma. Transl Lung Cancer Res 2018;7:189-98.

31. ClinicalTrials.gov A Feasibility Study Evaluating Surgery for Mesothelioma After Radiation Therapy Using Extensive Pleural Resection (SMARTER). Available online: https://clinicaltrials.gov/ct2/show/NCT04028570

32. Pehlivan B, Topkan E, Onal C, et al. Comparison of CT and integrated PET-CT based radiation therapy planning in patients with malignant pleural mesothelioma. Radiat Oncol 2009;4:35.

33. Allen AM, Czerminska M, Jänne PA, et al. Fatal pneumonitis associated with intensity-modulated radiation therapy for mesothelioma. Int J Radiat Oncol Biol Phys 2006;65:640-5.

34. Liao Z, Lee JJ, Komaki R, et al. Bayesian Adaptive Randomization Trial of Passive Scattering Proton Therapy and Intensity-Modulated Photon Radiotherapy for Locally Advanced Non-Small-Cell Lung Cancer. J Clin Oncol 2018;36:1813-22.

35. Popat S, Curioni-Fontecedro A, Polydoropoulou V, et al. A multicenter randomized Phase III trial comparing pembrolizumab versus standard chemotherapy for advanced pre-treated malignant pleural mesothelioma (MPM) -- results from the European Thoracic Oncology Platform (ETOP 9-15) PROMISE-MESO trial. Ann Oncol 2019;30 Suppl 5:931.

36. Xing D, Siva S, Hanna GG. The Abscopal Effect of Stereotactic Radiotherapy and Immunotherapy: Fool's Gold or El Dorado? Clin Oncol (R Coll Radiol) 2019;31:432-43.

37. ClinicalTrials.gov. Pembrolizumab and Hypofractionated Stereotactic Radiotherapy in Patients With Malignant Pleural Mesothelioma (MESO-PRIME). Available online: https://clinicaltrials.gov/ct2/show/NCT04166734

38. ClinicalTrails.gov. Atezolizumab, Pemetrexed Disodium, Cisplatin, and Surgery With or Without Radiation Therapy in Treating Patients With Stage I-III Pleural Malignant Mesothelioma. Available online: https:// clinicaltrials.gov/ct2/show/NCT03228537

39. Schröder C, Opitz I, Guckenberger M, et al. Stereotactic Body Radiation Therapy (SBRT) as Salvage Therapy for Oligorecurrent Pleural Mesothelioma After MultiModality Therapy. Front Oncol 2019;9:961.

Cite this article as: Hanna GG, John T, Ball DL. Controversies in the role of radiotherapy in pleural mesothelioma. Transl Lung Cancer Res 2021;10(4):2079-2087. doi: 10.21037/tlcr-20-583 